\title{
The Nuclear Starburst in NGC 4945
}

\author{
M. Wang \\ Max-Planck-Institut für Radioastronomie, Bonn, Germany \& P Purple \\ Mountain Observatory, Nanjing, China \\ C. Henkel \\ Max-Planck-Institut für Radioastronomie, Bonn, Germany \\ Y.-N. Chin \\ Dept. of Physics, Tamkang University, Taipei, Taiwan
}

J. B. Whiteoak

Australia Telescope National Facility, Epping, Australia

M. Hunt Cunningham

New South Wales, Australia

R. Mauersberger

IRAM, Avda. Divina Pastora, Granada, Spain

\begin{abstract}
NGC 4945 is with $D \sim 3-4$ Mpc one of the nearest starburst galaxies known and a goldmine for molecular cloud research. A multi-line mm-wave study has been carried out towards its nuclear region with the Swedish-ESO Submillimetre Telescope (SEST). The study covers the frequency range from $82 \mathrm{GHz}$ to $354 \mathrm{GHz}$ and includes 80 transitions of 19 molecules, including rare isotopebearing species. Applying a Large Velocity Gradient (LVG) code to the data, $\mathrm{H}_{2}$ densities and column densities of 22 molecular species are calculated. Many of these species indicate the presence of a prominent high density interstellar gas component characterized by $n_{\mathrm{H}_{2}} \sim 10^{5} \mathrm{~cm}^{-3}$. Abundances of molecular species are calculated and compared with abundances observed toward the starburst galaxies NGC 253 and M 82 and galactic sources. Apparent is an 'overabundance' of HNC and CN in the nuclear environment of NGC 4945. NGC 4945 is the second known starburst galaxy with an $\mathrm{HNC} / \mathrm{HCN}$ abundance ratio $\geq 1$. Carbon, nitrogen, oxygen and sulfur isotope ratios are also determined. The data indicate that high ${ }^{18} \mathrm{O} /{ }^{17} \mathrm{O}$, low ${ }^{16} \mathrm{O} /{ }^{18} \mathrm{O}$ and ${ }^{14} \mathrm{~N} /{ }^{15} \mathrm{~N}$ and perhaps also low ${ }^{32} \mathrm{~S} /{ }^{34} \mathrm{~S}$ ratios $(6.4 \pm 0.3,195 \pm 45,105 \pm 25$ and $13.5 \pm 2.5$, respectively) are characteristic properties of a starburst environment in an advanced evolutionary stage.
\end{abstract}

